Musées, Patrimoine et Culture scientifiques et techniques

$121 \mid 2009$

janvier - février 2009

\title{
Quand l'écologie devient objet(s) de musée
}

Marie Robert et Mathilde Leroux

\section{OpenEdition}

Journals

Édition électronique

URL : http://journals.openedition.org/ocim/269

DOI : $10.4000 /$ ocim.269

ISSN : 2108-646X

Éditeur

OCIM

Édition imprimée

Date de publication : 1 janvier 2009

Pagination : 19-27

ISSN : 0994-1908

Référence électronique

Marie Robert et Mathilde Leroux, «Quand l'écologie devient objet(s) de musée », La Lettre de l'OCIM [En ligne], 121 | 2009, mis en ligne le 01 janvier 2011, consulté le 10 décembre 2020. URL : http://

journals.openedition.org/ocim/269; DOI : https://doi.org/10.4000/ocim.269 


\section{Quand I'écologie devient objet(s) de musée}

\section{Marie Robert et Mathilde Leroux *}

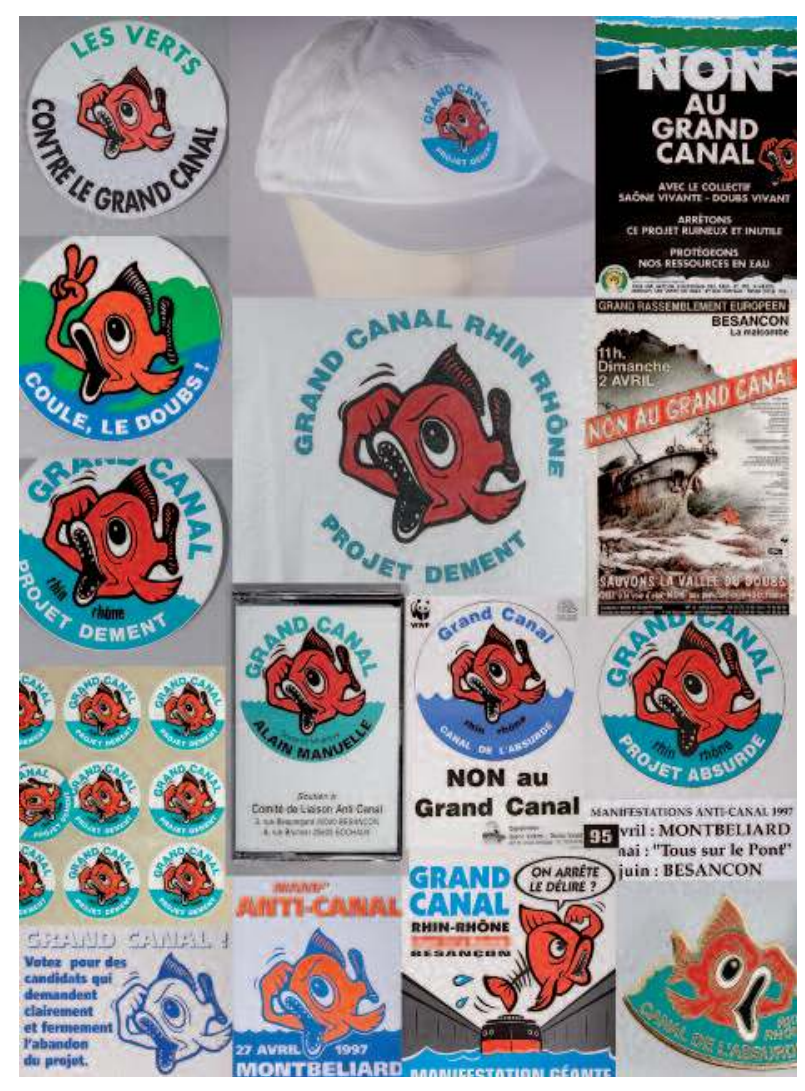

Autocollant, affiche, casquette, 1975-1997, don Pierre Parreaux et Gilles Sené (c) MuCEM/Christophe Fouin

* Marie Robert est conservatrice du patrimoine et a coordonné le programme muséographique sur le thème de l'Eau au MuCEM (2004-2008) marie.robert@culture.gouv.fr Mathilde Leroux, diplômée en Master 2 Mise en valeur et Gestion du patrimoine industriel, scientifique et technique, a été chargée d'enquête et de collecte au MuCEM en 2006
En dressant le bilan d'une campagne $d^{\prime}$ enquête et de collecte d'objets et de documents témoignant de pratiques contemporaines autour de l'eau menée par le musée des Civilisations de l'Europe et de la Méditerranée, les auteurs montrent comment les questions environnementales, le développement durable... sont devenus des sujets de préoccupation incontournables pour le musée de science et de société du XX|e siècle.

Dès 1992, des muséologues français et québécois dressaient, dans l'ouvrage, L'environnement entre au musée, un bilan de la prise en compte des thématiques environnementales dans les musées d'Histoire naturelle et de société (1). En vue de l'ouverture prochaine à Marseille du premier musée français de société et de civilisation consacré à l'Europe et à la Méditerranée, depuis l'an Mil jusqu'à nos jours, le MuCEM prépare un parcours de « référence » posant la question des rapports de l'homme à son environnement. Une exposition évolutive traitera de l'historicité et de la diversité des partis pris culturels par rapport à la nature, représentée de la manière la plus spectaculaire par l'eau ${ }^{(2)}$. La maîtrise et la répartition de cette ressource sont en effet au cour de l'organisation des sociétés, et elle est au centre de relations de coopération et de conflits entre les sociétés.

Le musée présentera des œuvres d'art aussi bien que des objets de la vie quotidienne, des sons et des images animées, provenant d'époques et de lieux contrastés, en les faisant dialoguer, afin de faire percevoir 
aux publics les parentés en même temps que la diversité des cultures matérielles et immatérielles de l'espace euro-méditerranéen (3). Les riches collections issues des fonds de l'ancien musée national des Arts et Traditions populaires (MNATP) et de l'ancien département Europe du musée de l'Homme restent pourtant lacunaires et doivent être complétées par des objets témoignant de pratiques contemporaines, dans des champs jusque-là peu développés : économie, enjeux politiques, urbanisme, travaux hydrauliques, confort sanitaire, environnement.

En dépit des profondes inégalités qui persistent quant à l'accès et au partage d'une ressource convoitée et disputée - l'eau -, les sociétés européennes sont traversées aujourd'hui par une préoccupation commune d'écologisation de la pensée et des pratiques relatives aux usages de l'eau, les plus intimes comme les plus collectifs (4). La protection de l'environnement naturel et de la ressource aquatique devient une priorité : souci pour la qualité de la boisson, intérêt pour la survie des écosystèmes et de la biodiversité, crainte de la raréfaction de l'eau douce, constat que les solidarités s'émoussent face aux transformations rapides de la qualité disponible. Si à partir du XIXe siècle commençait à se fissurer la conception euphorique du pouvoir de l'homme occidental sur la nature, quand les premiers effets de l'industrialisation devinrent visibles, ce n'est que récemment, après la période des Trente Glorieuses (1945-1975), que s'est opérée la prise de conscience d'une altération irréversible de l'eau dans les régions industrialisées (5). Même si gaspillages et pollutions sont anciens, un imaginaire d'aspiration à une eau " sauvage ", à la fois authentique et protégée, se construit depuis les années 1970, quand apparaissent constat de l'altération de la nature et critique du modèle productiviste.

Dans cette perspective, une équipe de conservateurs et de chercheurs guidée par un conseil scientifique (6) a mené une campagne d'enquête et de collecte, afin de témoigner de la multiplicité des acteurs engagés dans le phénomène écologique, de repérer motivations et modalités d'action, bref d'évoquer les dimensions sociales, culturelles et politiques de l'environnement, vécu et construit par les sociétés de l'Europe et de la Méditerranée, à travers des productions matérielles fragiles et éphémères. La perspective comparatiste a guidé cette collecte portant sur 24 pays différents, les enquêtes de terrain nous ayant conduits à Besançon, Coutances, Biarritz, Brest, Paris, Marseille mais aussi à Zurich,
Amsterdam, Zagreb, Lisbonne, Saragosse, Athènes, Amsterdam et Tunis. Menée sur six mois la campagne a permis d'acquérir près de 2000 objets et documents : affiches, autocollants, pancartes, banderoles, cartes et prospectus, vêtements, badges, magnets et porte-clefs, journaux et revues, jeux et jouets, produits ménagers et mobilier sanitaire, bouteilles d'eau, dessins, dossiers de presse, et autres documents d'information...

\section{L'enquête et la collecte}

\section{Méthodologie}

Les canaux par lesquels le musée enrichit son patrimoine sont variés : enquêtes de terrain, opérations de sauvetage, collectes plus ponctuelles (auprès d'antiquaires ou de particuliers, en ventes publiques...), ainsi que campagnes thématiques et comparatives. Celles-ci sont des collectes raisonnées et systématiques permettant l'acquisition d'ensembles documentés. Leur principe s'est imposé au MNATP dès les années 1970. Il constitue aujourd'hui encore l'une des originalités majeures de la politique de recherche et d'acquisition de l'établissement. La campagne repose sur un pré-financement global par la Réunion des Musées nationaux sur un projet thématique d'une durée d'une année ou plus. La démarche d'enquête s'appuie sur une méthodologie rigoureuse, dont Marcel Maget avait théorisé les fondements dans son Guide d'étude des comportements culturels ${ }^{(7)}$. En amont, un travail approfondi de documentation bibliographique est réalisé, ainsi

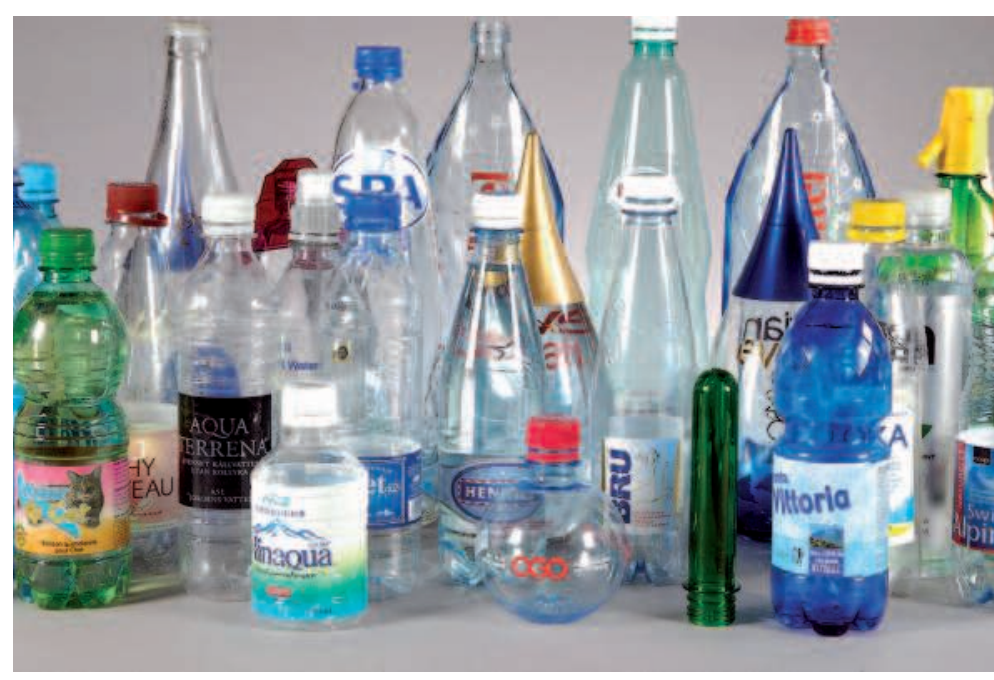

Un échantillon des 400 bouteilles d'eau collectées (๑) MuCEM/Christophe Fouin 
que des recherches au sein des collections publiques françaises. Les informations recueillies par voie d'entretien sont ensuite analysées. Ces dernières années, les campagnes ont concerné des thèmes aussi divers que "les chemins des musiques électro-amplifiées ", "faire la crèche », le sida, le graff-tag, l'artisanat du verre, le mariage... (8)

Documentés par des entretiens, des photographies et des films, les objets acquis lors de notre campagne manifestent le développement et la généralisation d'une conscience environnementale et l'émergence de valeurs écologistes résultant de la relégation des besoins économiques et sécuritaires au second rang des sociétés d'abondance, de l'accroissement des ressources culturelles et sociales des individus (les plus diplômés sont aussi les plus environnementalistes), mais aussi de différentes attitudes liées à des spécificités culturelles, politiques (rapport à l'État) ou religieuses (protestantisme vs catholicisme par exemple). Ces objets témoignent de groupes qui s'organisent, depuis la manifestation spontanée et l'association locale jusqu'à l'engagement politique, pour repenser et se réapproprier la ressource aquatique. Économie (répartir la quantité de l'eau disponible), écologie (gérer la qualité), patrimonialisation (redécouvrir, revaloriser et réinventer des pratiques plus ou moins ancestrales) sont les maîtres mots de cette évolution.

\section{Quels objets et quelle légitimité ?}

Objets banals, contemporains, sériels, ces objets collectés sont aussi des objets qui disparaissent aussi vite qu'ils ont été produits, du fait de leur rapide obsolescence fonctionnelle et matérielle, des objets dont l'intérêt ne surgit parfois qu'avec le recul du temps, mais des objets qui tissent des réseaux de sens autour des similarités et des différences, des emprunts et des confrontations d'un espace social à l'autre. Que peuvent-ils alors dire sur les pratiques et les symboliques auxquelles ils sont attachés, que peut en faire le musée, comment peut-il les exposer? Ainsi les traces des premières expressions de l'écologie politique (affiches, archives) ont été collectées par quelques institutions précurseurs : Bibliothèque de documentation internationale contemporaine, musée du Vivant, musée de Bretagne, Archives nationales. Celles-ci conservent l'une des premières affiches de René Dumont qui détourne « La Parabole des aveugles », scène du Nouveau Testament peinte par Pieter Brueghel l'ancien en 1568.

Ces objets « modestes », que les musées en région et les archives ont depuis longtemps commencé à collecter ('exposition Rester libres ! en 2007-2008 du musée Dauphinois se terminait par une série d'affiches évoquant les luttes féministes, ouvrières et... écologiques qui ont traversé l'histoire dauphinoise les cinquante dernières années), trouvent aujourd'hui encore difficilement leur légitimité aux yeux des instances muséales parisiennes (9).

Certains documents nous semblent déjà anciens, comme la quarantaine d'affiches recueillies au siège des Verts parisiens et auprès du comité de Liaison Anti-canal de Besançon. Elles rappellent que les luttes de terrain constituèrent d'abord l'essentiel de l'action dans les années 1970 (au moment où les écologistes se présentent à différentes élections locales 10) : contre le nucléaire civil (Fessenheim et le Bugey en 1971, Plogoff à partir de 1975) et militaire (Larzac dès 1971, Creys-Malville en 1976) ; contre les pollutions maritimes (Amoco Cadiz en 1978) ; contre des chantiers hydrauliques destructeurs de paysages et de sites urbains (tel le projet de canal Rhin-Rhône lancé en 1975). Ainsi le programme électronucléaire, en 1974, rassemble contre lui

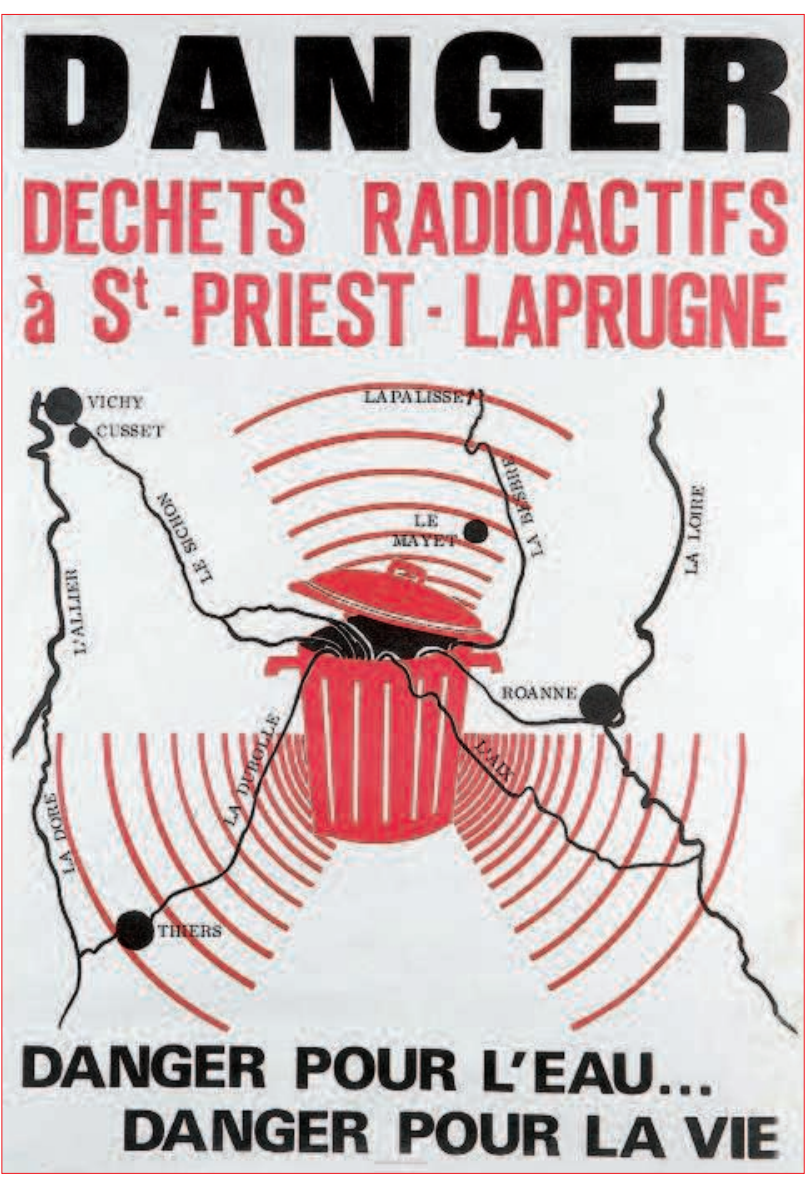

Danger déchets radioactifs, affichette, Le Mayetde-Montagne (France), vers 1980-1984, don Les Verts (c) MuCEM/Christophe Fouin 
universitaires, consommateurs militants, agriculteurs et adhérents de comités de quartiers. Une explosion associative s'exprime alors par des manifestations souvent spectaculaires. Ainsi, en 1955 avait débuté l'exploitation, prévue pour quinze ans, d'une mine d'uranium à Saint-Priest-Laprugne, à la limite de l'Allier, de la Loire et du Puy-de-Dôme. Lorsqu'un projet de stockage des déchets radioactifs sur le site est envisagé en 1979, une partie des élus et de la population locale s'y oppose activement. Durant cinq ans, la mobilisation dans cette région est sans précédent pour un problème environnemental. Le projet est abandonné en 1984, malgré le soutien des « prugnards », ces mineurs qui souhaitent conserver une activité industrielle pourvoyeuse d'emplois et d'activités. La pollution de l'eau est alors perçue comme un risque écologique majeur. Réalisée pour l'occasion, l'affiche, au graphisme élémentaire comme nombre des visuels des Verts (du soleil rouge des autocollants anti-nucléaires au tournesol emblématique du parti) participe de l'histoire des premières luttes contre l'atome, caractérisées alors par la convocation de visions souvent macabres et répulsives ${ }^{(11)}$.

Mais d'autres objets qui, pour leur producteurs, ont vocation à être immédiatement efficaces, ont-ils leur place dans une campagne comme celle-ci et doiventils figurer définitivement à l'inventaire d'un musée ? Ainsi, une centaine d'objets a été collectée auprès de la Plataforma en Défensa de l'Ebre, incarnation associative d'un mouvement né dans les années 1970 pour protéger l'Ebre, le plus puissant fleuve d'Espagne, des aménagements visant à redessiner la carte hydraulique du pays. Il a pris de l'ampleur et s'est constitué officiellement en ONG en 1995, sous le

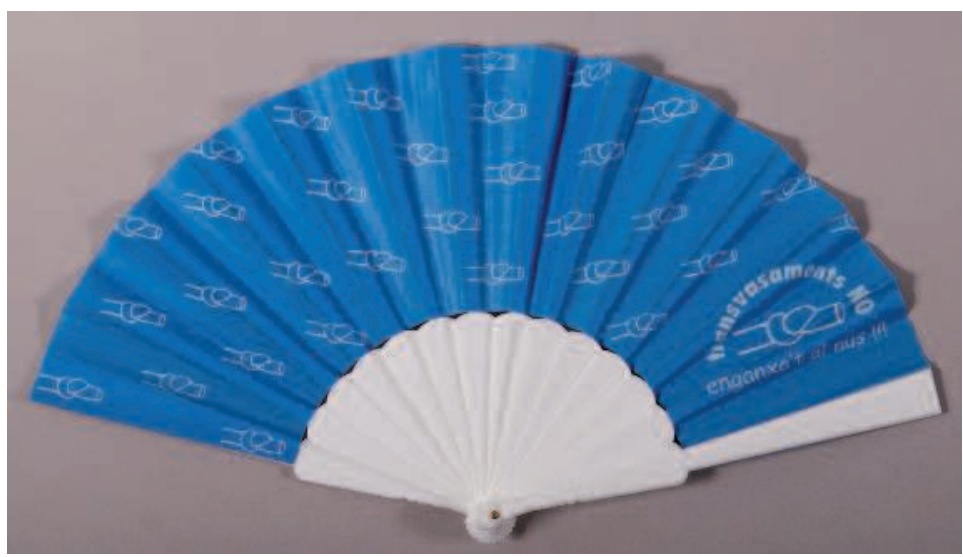

Transvasaments No/enganxa't al nus !!!, éventail, Tortosa (Espagne), 2000-2004 ๑) MUCEM/Christophe Fouin nom de Coordinadora Anti-Transvasament. Afin de financer et de rendre visible son action, il a émis une série d'objets portant tous le même symbole du tuyau noué, vendus notamment au marché couvert de Tortosa et lors de manifestations.

C'est aussi dans la comparaison qu'un éventail collecté trouve son sens : nous avons pu constater que toutes les associations rencontrées produisent ce type d'objet massivement distribué (briquet, casquette, cartes postales, autocollants) dont seuls l'effigie ou le motif stylisés varient. Et l'on voit que si la lutte environnementale demeure le socle de référence pour tous ces mouvements, ce type d'objet nous permet aussi de lire les préoccupations nationales spécifiques : dépérissement des forêts en Allemagne de l'Ouest, pollution du Rhin et montée de la mer aux Pays-Bas, plantations industrielles au Portugal, collecte sélective en Italie ou recyclage des déchets en Suisse.

\section{Interlocuteurs}

Un nombre croissant d'acteurs sociaux produisant de « l'idéologie » environnementaliste, nous avons choisi de nous intéresser principalement à des agents producteurs de discours et de normes, prescripteurs de comportements. En effet la transmission des normes et des valeurs et la socialisation des individus ne passent plus autant qu'autrefois par le contact direct (famille, pairs, écoles, instances religieuses, appartenances associatives et politiques), mais se réalisent également par une profusion d'injonctions médiatiques (télévision, Internet, presse, affichage, radio) qui investissent les espaces publics et privés, et donnent aux prescripteurs une présence massive. Deux types d'acteurs ont été sollicités : les associations d'une part, et ces prescripteurs institutionnels et économiques que sont ministères, agences de l'eau, sociétés de distribution, entreprises commerciales, d'autre part.

\section{Du coté du militant : objets politiques}

Au travers des enquêtes menées, nous avons interrogé le « fait » militant, la façon dont il se construit et évolue, ainsi que les pratiques qu'il induit. Les objets collectés matérialisent diverses formes d'actions : réunions publiques, activités pédagogiques, actions médiatiques, expertises scientifiques, manifestations, sit-in, pétitions, appels au boycottage ou actions en justice.

Trois types d'objets témoins ont été recueillis. Ils illustrent avec force la circulation des modes opératoires du militant « européen ». Il s'agit d'une part 


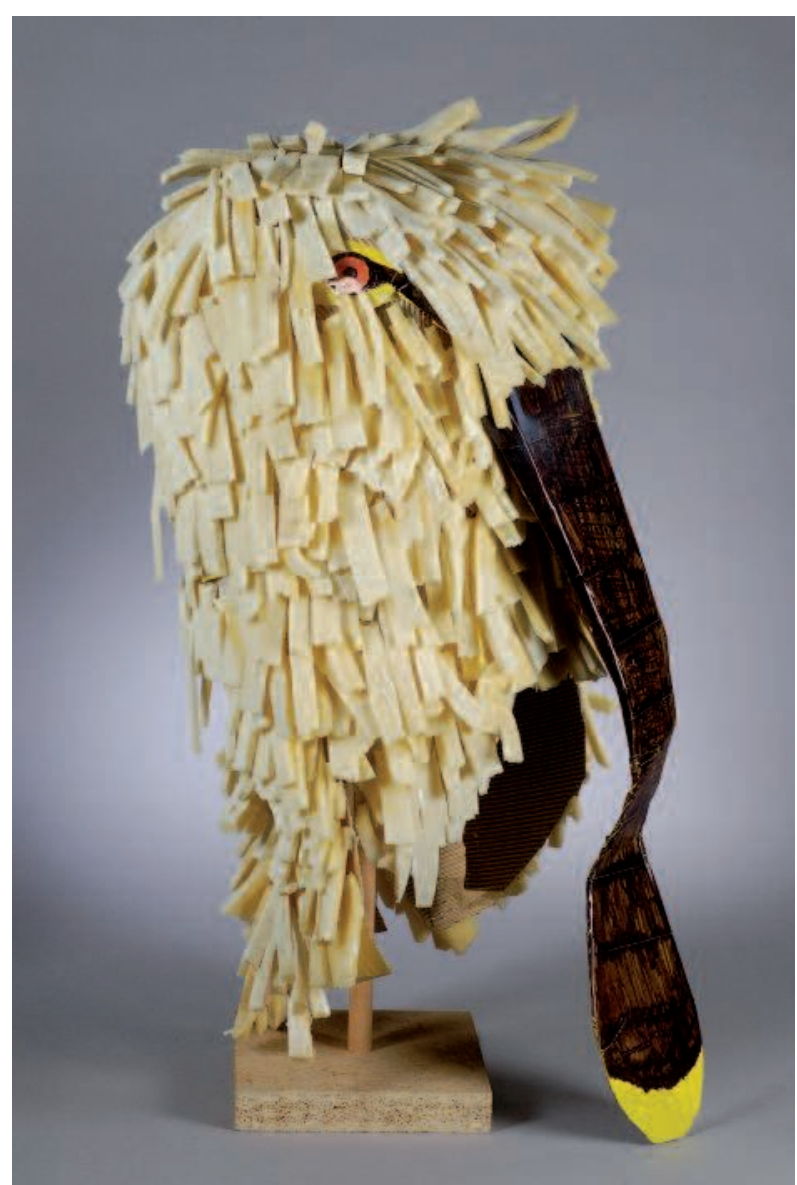

Masque de spatule, Zagreb (Croatie), 2004, don Irma Popovic (c) MuCEM/Christophe Fouin

d'objets de manifestation, souvent spectaculaires : fûts de déchets radioactifs transformés en tambour de rue des militants de l'ONG Greenpeace, radeau flottant constitué de bouteilles d'Eaux et Rivières de Bretagne, banderoles gigantesques, autocollants, panneaux pour défilés. À cette catégorie appartient un masque, provenant de l'ONG croate Zelena Akcija (Action verte). Il représente une spatule blanche, oiseau de la famille du héron, habitant dans les zones humides côtières de l'Adriatique, et menacé par la disparition des habitats de nidification et d'alimentation. Il a été porté dans le cadre de la campagne «Sauvons la rivière Drava », lancée fin 2003 contre le projet de construction d'une centrale hydroélectrique. Déguisés en poissons et en animaux d'eau douce, portant des banderoles (" Nous aussi avons besoin d'un endroit pour vivre »), les manifestants ont parcouru les rues de Zagreb.

Nous avons également collecté des objets "identitaires ", qui permettent de financer le fonctionnement de l'organisation. Manufacturés ou fabriqués par les militants eux-mêmes, ils portent le symbole du mouvement (pins, cartes postales, casquettes, drapeaux, tee-shirts). Le vêtement militant est souvent gardé comme souvenir et commémoration d'un engagement ou d'un évènement. Ainsi ont été rassemblés plus de cent objets déclinant le motif de poisson qui a symbolisé, de 1975 à 1997, la lutte menée par un collectif d'associations contre le projet de mise à grand gabarit du canal Rhin-Rhône. Visant à relier le nord de l'Europe à la Méditerranée, celui-ci a suscité de vives réactions de contestation. Tandis que la canalisation à grand gabarit du Rhône puis de la Saône s'est achevée en 1980, le mouvement des opposants à la réalisation de la liaison Saône-Rhin s'est intensifié, aboutissant à son abandon en 1997. Ce mouvement associatif, très organisé, a exploré des formes d'action festive : concerts, appel à coupure de courant, concours de pêche, manifestations, expositions d'artistes. Afin de financer réunions, supports d'informations et déplacements, il a mis en vente une série d’objets variés ornés du logo dessiné par Jean Parreaux. Investi d'une dimension symbolique de sauveur et de révélateur, qui lui est attribuée dans différentes religions et mythologies, le poisson, motif récurrent dans l'espace européen, jaillit des eaux, furieux, pour mobiliser l'opinion publique contre le projet.

Enfin divers objets de sensibilisation ont été réunis : matériel didactique (jeux de société, cartes, livrets, instruments de mesure de la pollution) ou objets de promotion (brochures, revues, autocollants, tableaux), émis à destination des scolaires, des citoyens, des consommateurs.

Une planche de surf décorée de déchets récoltés sur la plage (produits d'entretien, jouets, aérosols, mégots) a été produite par l'association Surfrider Foundation, collectif d'usagers de la mer et de la plage, dont Tom Curren, triple champion du monde de surf. Ce montage-collage participe des moyens d'alerte auprès de l'opinion publique et des élus afin de modifier les comportements vis-à-vis du littoral. Avec ce surf, pilier de tous les stands présents à l'occasion des compétitions, des actions pédagogiques et des expositions depuis 1990, l'art rencontre l'éducation à l'environnement. Le recyclage se trouve au cœur du processus de création, démarche de récupération qui emprunte à l'Arbre de Martial Raysse (1960), aux Tableaux-pièges de Spoerri (1963), aux Poubelles d'Arman (1971), et plus encore aux gestes militants de la nouvelle génération de la scène artistique, que ce soit Gilles Clément avec son $M a-$ nifeste du Tiers Paysage (2007) ou Stuart Haygart et son Tide Chandelier (1996), luminaire composé lui aussi d'objets ramassés sur les plages du Kent, récemment exposé aux Galeries nationales du Grand Palais (12). 


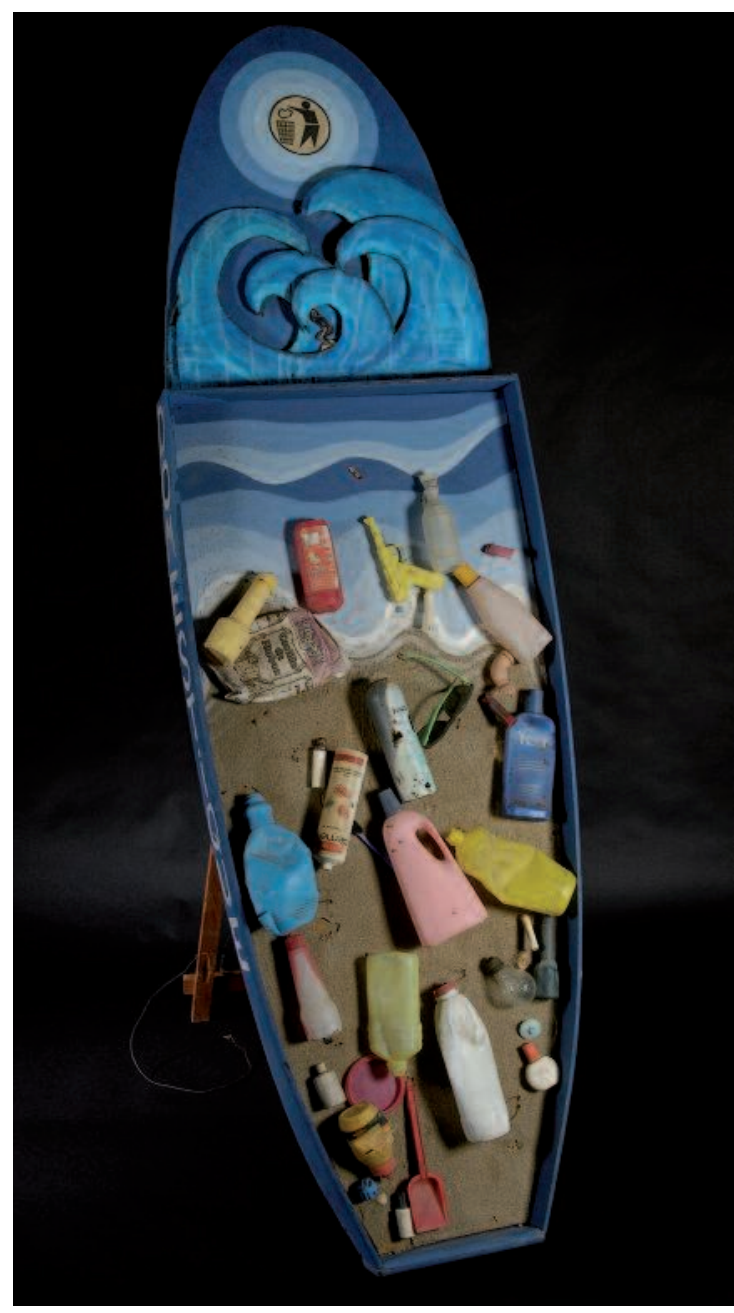

Totem sur la durée de vie des déchets, Biarritz (France), années 1990 ๑) MuCEM/Christophe Fouin

L'enquête et la collecte auprès de ces organisations ont permis d'observer des évolutions dans les formes d'engagement et dans la sociologie des militants, dans les formes d'action et de structuration, dans le rôle joué entre pouvoirs publics et société civile.

Parmi les associations étudiées, certaines s'institutionnalisent progressivement, comme Eau et Rivières de Bretagne ou Stichting Reinwater. À force d'études et d'expertises, mais aussi d'actions d'éducation à l'environnement, elles ont peu à peu acquis une légitimité faisant d'elles des interlocuteurs respectés par les pouvoirs publics. Leurs responsables, spécialistes reconnus (universitaires, ingénieurs), sont souvent consultés par les autorités avec lesquelles ils agissent en concertation et dont ils sollicitent la reconnaissance, notamment à travers l'obtention d'agréments. Cependant, cette reconnaissance contribue à minimiser le caractère marginal et contestataire originellement propre aux associations éco-environnementales (13).
Le militantisme classique est progressivement délaissé. Notre collecte témoigne d'actions de plus en plus festives, familiales, ludiques, visibles (créer un évènement, former des collectifs), volontairement optimistes et plus médiatiques, où l'imagination et la créativité cherchent à toucher les sensibilités (14).

Cette évolution est visible pour l'organisation néerlandaise Stichting Reinwater ("Fondation pour une eau pure »), fondée en 1974. Ses premières manifestations, des recours en justice et des actions concrètes, visent à dénoncer les pollutions des eaux générées par l'industrie. À partir des années 1990, elle réoriente ses missions vers la gestion durable de l'eau, en développant des activités beaucoup plus pédagogiques, de sensibilisation et de formation, en relation avec les pouvoirs publics. Aujourd'hui son action s'inscrit également dans le cadre de réseaux internationaux dont Rhinenet-Réseau rhénan, Mosa Nostra et European Rivers Network. Sa revue, dont nous conservons une série de numéros, témoigne de la transformation de la fondation entre 1981 et 2006, tant dans son mode d'action que dans son discours. Les premières couvertures, en noir et blanc, annonçaient d'austères analyses d'échantillons d'eau polluée et des reportages photographiques sur les zones concernées. Aujourd'hui, les couvertures montrent des enfants joyeux participant aux activités pédagogiques. Le discours alarmiste des années 1970-1980 a fait place à une démarche didactique, visant à former le citoyen de demain.

En quarante ans l'action associative a fait surgir la question environnementale sur la scène politique en amenant les citoyens à s'intéresser concrètement aux affaires publiques, à intégrer les idées et les propositions des militants. "Par ses démarches, pétitions, distributions de tracts, diffusion de bulletins, appels aux médias, défilés ludiques ou manifs, l'immense nébuleuse associative, du moindre comité aux plus grandes organisations, a collectivement éveillé l'opinion. Longtemps méprisée, et même vilipendée, par les acteurs économiques, elle a fini par les impressionner ne serait-ce que par le nouveau marché que représentent ses militants. À l'égard de l'administration, y compris celle de l'environnement elle a joué le rôle d'aiguillon, l'obligeant souvent à "faire son métier" » (15). Les organisations de cette nébuleuse sont devenues des partenaires indispensables dans la conception et dans la mise en œuvre des politiques environnementales. 


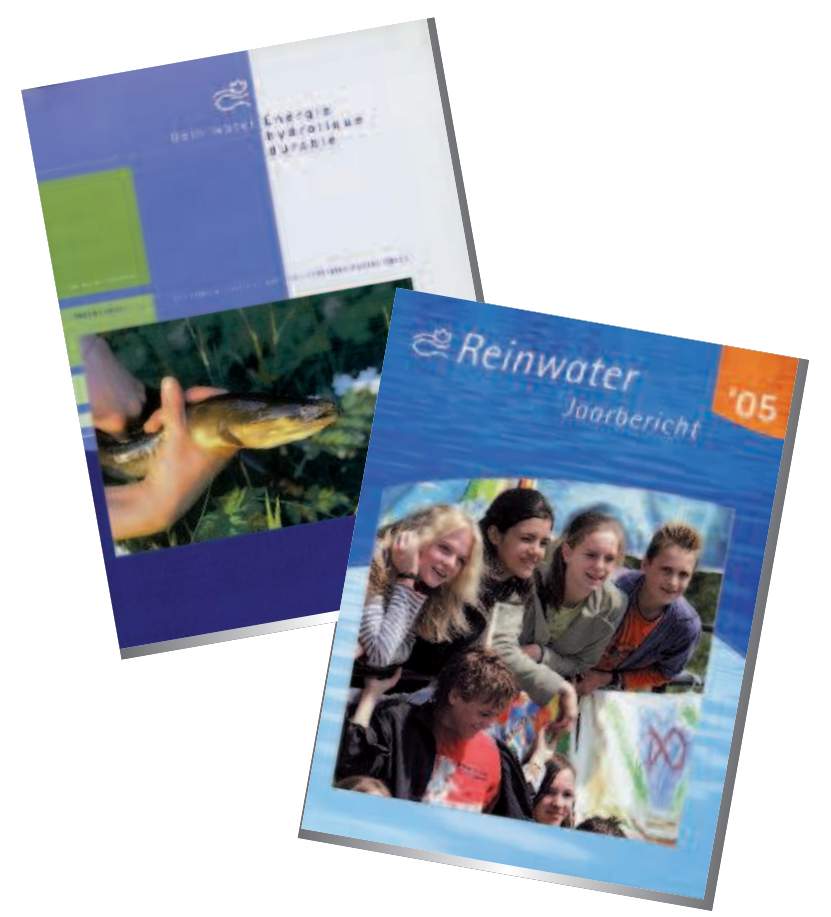

Revues Reinwater/Reinwasser et Reinwater Jaarbericht, 1981 et 2006, don Stichting Reinwater ( ) MuCEM/Christophe Fouin

\section{Du coté du producteur de biens et de services : les objets prescripteurs}

La protection de l'environnement et le développement durable sont devenus des thèmes omniprésents en Europe occidentale (16). Une part croissante des consommateurs européens, et plus seulement l'ancienne génération militante, s'alimente et s'habille, entretient son corps, sa maison et son jardin en se souciant du devenir de l'espèce humaine, du monde animal et des ressources naturelles. Nombre de secteurs de la vie économique et sociale se sont mis à cette vague verte : littérature, urbanisme, transports, mode, mobilier, jardinage, sexualité, alimentation, banque, cosmétique, agriculture, technologies de l'information, médication, coiffure et même choix du prénom intègrent désormais des préoccupations écologiques. De la petite et moyenne entreprise à la multinationale, l'argument écologique est au cœur des stratégies industrielles et commerciales.

Si nous avons collecté objets et documents provenant des champs concernés par la question de l'eau (transport collectif, architecture durable, tourisme, grande distribution, boisson), nous avons privilégié quelques acteurs que l'on pouvait comparer sur l'espace euro-méditerranéen : collectivités publiques (ministères de l'Environnement, municipalités, syndicats de commune), agences de l'eau, ainsi que sociétés de distribution et de production d'eau embouteillée. Où l'on constate que les frontières entre institutionnels et industriels sont plus floues qu'il n'y parait.

Sensibiliser, informer

et éduquer le consommateur

Nombre d'agences et d'entreprises de distribution et de traitement de l'eau mettent en place des stratégies de communication visant à sensibiliser, informer et éduquer le consommateur sur la préservation des milieux aquatiques, en développant des outils de diffusion (affiches, contenants, objets dérivés, jeux) à destination de « publics-cibles ».

Ainsi chaque année depuis 1997, le service municipal d'assainissement de l'eau (SMAS) de la mairie d'Almada, commune limitrophe de Lisbonne, édite une affiche dédiée à la journée nationale de l'Eau, le $1^{\text {er }}$ octobre, et désormais une affiche pour la journée mondiale de l'Eau, le 22 mars. Avec un trait sobre et stylisé, une combinaison de couleurs soulignant la fertilité de l'eau, des motifs simples (faune et flore principalement), le sculpteur et illustrateur João Machado, né en 1942 et l'un des graphistes les plus célèbres de son pays, exprime toutes les vertus bénéfiques de l'eau. Arbre de vie, eau de rosée, moulin, colombe, poisson, arc en ciel... sont des symboles qui empruntent au répertoire chrétien et plus largement populaire et qui sont facilement partagés par le grand public.

Ces stratégies de communication visent tout particulièrement les enfants en milieu scolaire, à travers des objets traitant de façon ludique des enjeux de l'eau : dossiers à destination des enseignants, jeux de société, mallettes pédagogiques. Outils interactifs, alliant jeu, expériences visuelles, signification morale et rôle éducatif, ils permettent d'introduire le débat et de travailler en groupe. Plus largement, support de significations, mode de transmission des représentations légitimes produites par les adultes, le jouet est de plus en plus un médiateur d'éléments culturels. Trois des jeux collectés sont inspirés par le jeu de l'oie : le « Jeu de Lola », diffusé par le groupe Eaux de Marseille, le « Jeu de l'eau », conçu par l'agence de l'eau Rhône-Méditerranée-Corse (en matières recyclées et recyclables), ainsi qu'un jeu de sol produit par le service municipal d'assainissement de l'eau d'Almada (17). Le jeu de l'oie comporte un certain nombre de pièges, ici remplacés par des questions ou des illustrations relatives à l'eau : le cycle de l'eau, les éco-gestes, les propriétés de l'eau, la connaissance des milieux aquatiques, les inégalités d'accès à l'eau, la solidarité internationale... Parcours individuels d'apprentissage au comportement éco-citoyen, ces jeux visent à faire prendre conscience aux enfants de leur intérêt commun. 


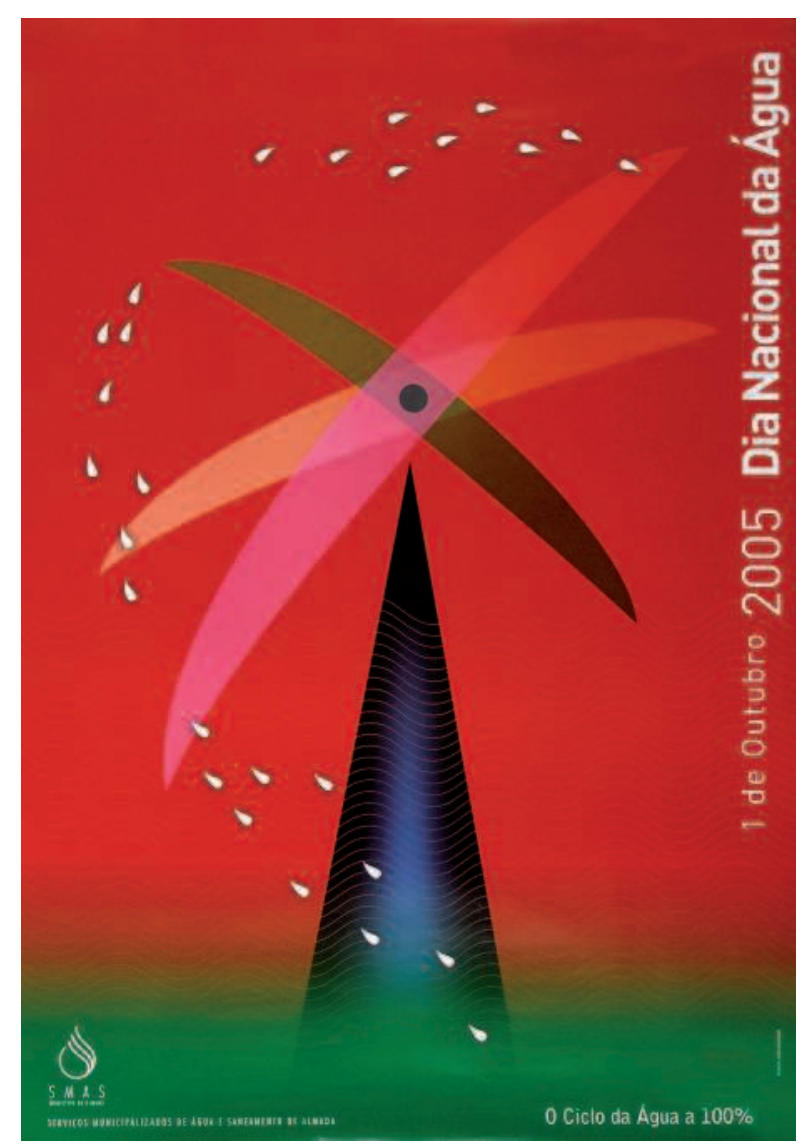

Affiche de João Machado (Portugal), 2005, don SMAS () MuCEM/Christophe Fouin

L'oie est, dans chacun de ces cas, remplacée par une goutte d'eau personnifiée. Ce jeu qui s'inspire très fréquemment des grands évènements historiques et des sujets d'actualité, est un bon indicateur de leur prise en compte par le corps social. Que l'on songe aux nombreux jeux de l'oie qui ont eu pour thème l'Affaire Dreyfus (18).

\section{Contenu et contenants :}

\section{les modes de consommation}

Par ailleurs, le développement du marché de l'eau en bouteilles a connu en Europe une formidable expansion liée à deux phénomènes : la suspicion des consommateurs à l'égard de l'eau du robinet et la recherche croissante d'aliments «santé». Boire une eau, c'est s'approprier ses bienfaits, subir ses méfaits, réels ou supposés. À travers l'eau que l'on boit, c'est aussi l'essence de la nature que l'on incorpore dans sa dimension magique. D'où l'obsession du label, de l'étiquetage, de la garantie des origines (19).

Nous avons ainsi constitué une collection d'étude d'environ 400 bouteilles. Ces objets issus de l'industrie revêtent un caractère banal et éphémère. Cependant, n'est-ce pas la quantité de bouteilles recueillies qui en fait l'intérêt, permet étude et comparaison ? Le vertigineux renouvellement des bouteilles en matières plastiques comme en verre, qui jouent sur la forme, la couleur, l'étiquetage, l'édition en séries limitées, festives ou évènementielles nous enjoint de mener une archéologie du contemporain qui s'attacherait aux techniques, aux matériaux comme aux signifiants des contenants.

En réaction à ce phénomène de consommation, plusieurs sociétés de distribution en France et en Europe ont mis en place des campagnes de communication pour valoriser auprès des consommateurs la qualité de l'eau du robinet et les encourager à baisser leur consommation d'eau embouteillée. L'eau du robinet ne bénéficiant pas d'un contenant aussi identifiable que la bouteille, "Eaux de Marseille », «Eaux de Paris » et le Syndicat des Eaux d'Île-de-France, entre autres, ont fait appel à des designers pour la conception de carafes en verre. En 1999, le SEDIF annonce son nouveau procédé de traitement de l'eau par nanofiltration en diffusant largement sa nouvelle carafe auprès des habitants des 39 communes de la région parisienne alimentées par l'Oise. En collaboration avec le centre international de recherche sur le Verre et les Arts plastiques, "Eaux de Marseille » et "Eaux de Paris » ont sélectionné deux designers, Sylvain Dubuisson et Pierre Charpin, dont les créations archétypales trônent désormais sur les tables quotidiennes de milliers de citadins.

Sujet d'actualité polarisant fortement l'opinion, la préservation de l'environnement est aussi une question qui sourd depuis des décennies au cœur des sociétés occidentales, soulevant de nombreuses pistes de réflexion et d'interrogation : rôle du progrès technique et scientifique, urbanisation et croissance démographique, consommation de masse, rapport à la vie animale et végétale, distinction entre les temps de loisirs et les temps du travail, démocratie et participation citoyenne... Il est du rôle du musée de se confronter à ces questions, même si cela ne va pas de soi. "Car le musée n'a pas coutume de traiter de sujets qui appartiennent au présent et qui, de surcroît, demandent de prendre position. Il est plutôt tourné vers la conservation d'un passé dont la valeur sociale (esthétique, mémorielle, scientifique) est admise par tous ; bref, d'un patrimoine reconnu» (20).

Que conserver de la production sérielle des objets contemporains? Quelles traces garder des pratiques sociales et culturelles ordinaires, à la fois mondialisées et diversifiées ? Quels objets font sens (21) ? Cette campagne souhaite par ses résultats et ses 


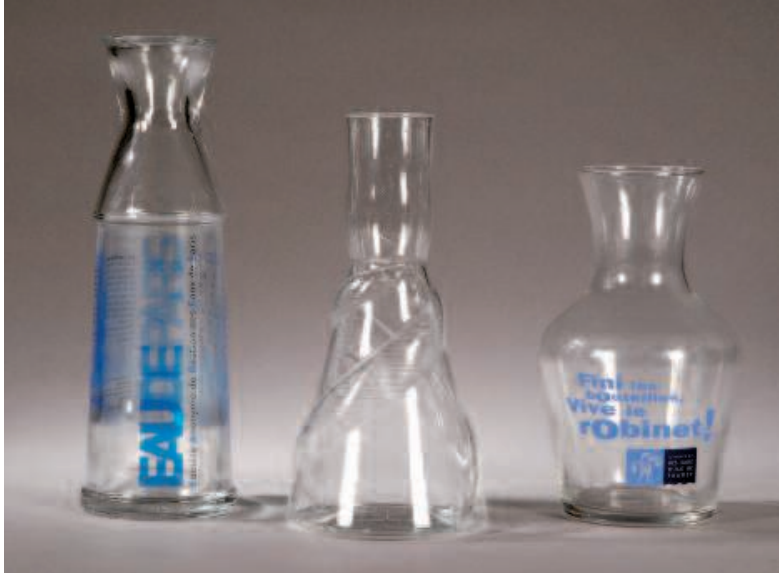

Eau de Paris, Eaux de Marseille et Fini les bouteilles, vive le robinet !, carafes de Pierre Charpin, Sylvain Dubuisson et anonyme, France, 1999-2004, dons de la société anonyme de gestion des Eaux de Paris, de la société des Eaux de Marseille et de Pascale Schmitt ๑) MUCEM/Christophe Fouin

questionnements contribuer à consolider les bases méthodologiques et épistémologiques de la collecte du contemporain et de la constitution de collections pour un musée d'Anthropologie.

Les auteurs tiennent à remercier pour leur collaboration les membres du conseil scientifique, les personnes qui les ont fait bénéficier de leurs réflexions et conseils (J. Païs de Brito, directeur du musée national d'Ethnographie à Lisbonne et F. Loux, directeur de Recherches honoraire au CNRS), les membres de l'équipe de travail (D.-M. Boëll, B. Rolland-Villemot, F. Wateau, F. Tamarozzi, E. Moreau, K. Rannou, P. Békus, C. Fouin, J. Albertini, D. Czarny, M. Zveguinzoff) ainsi que l'ensemble des interlocuteurs et donateurs pour le temps et la confiance qu'ils ont accordés, pour leur témoignage et pour les objets et documents qu'ils ont offerts au musée.

\section{Notes}

(1) Davallon, J., Granmont, G. et Schiele, B. L'environnement entre au musée. Presses universitaires de Lyon, musée de la Civilisation de Québec, 1992.

(2) Notre nature est historique et, à chaque période de l'histoire, nous constituons un état de nature spécifique. Voir Barrau, J. Les hommes dans la nature, esquisse d'une histoire naturelle des sociétés et des mœurs humaines, in Poirier, J. Histoire des moeurs, Les coordonnées de l'homme et la culture matérielle. Paris : Gallimard, 1990, tome 1, pp. 9-58.

(3) À ce sujet, voir Boëll, D.-M. Trésors du quotidien, du musée national des Arts et Traditions populaires au musée des Civilisations de l'Europe et de la Méditerranée. Paris : Réunion des musées nationaux, 2005.
(4) Kaloara, B. Le culte contemporain de la nature, in Gonseth M.-O., Hainard, J. et Kaehr, R. Natures en tête. Neuchâtel : Musée d'Ethnographie, 1996, pp. 85-109. Planchat, C. Protéger l'environnement : un objectif pour une grande majorité de Français, INSEE Première, janvier 2007. Roy, A. L'environnement, de plus en plus intégré dans les gestes et attitudes des Français, Institut français de l'Environnement, ${ }^{\circ} 109$, janvierfévrier 2006. Vers une consommation durable des ménages? Tendances et politiques dans les pays de l'OCDE. OCDE, 2002.

(5) Acot, P. Histoire de l'écologie. Paris : Presses universitaires de France, 1985. Jacob, J. Histoire de l'écologie politique. Paris : Albin Michel, 1999. (6) Composé de Fabienne Wateau, chargée de Recherches CNRS, laboratoire d'Ethnologie et de Sociologie comparative, Marie-Christine Grasse, conservateur en chef du musée international de la Parfumerie à Grasse, et de Torbjorn Bildgård, chargé de Recherches en Sociologie alimentaire (université de Sciences sociales de Stockholm/ EHESS).

(7) Civilisations du Sud, Paris, 1953.

(8) Sur les collectes au MuCEM, voir Boëll, D.-M. et Colardelle, M. Les collectes du musée des Civilisations de l'Europe et de la Méditerranée une perspective comparatiste, Museum, à paraître.

(9) Ces objets trop « modestes » ne trouvent pas leur place dans la Revue des musées de France, par exemple.

(10) Et l'agronome René Dumont devient le premier candidat écologiste à l'élection présidentielle en 1974 : au cours de la campagne télévisée, il vide un verre devant les caméras pour prévenir de la raréfaction de l'eau. (11) Gervereau, L. La Propagande par l'affiche. Alternatives, 1991.

(12) Design contre Design. Deux siècles de création, RMN, Paris, 2007. Et si la créativité populaire s'inspire des formes savantes de l'art, les emprunts circulent dans les deux sens : une série de banderoles syndicales a été exposée en septembre 2008 par Ed Hall au Palais de Tokyo dans l'exposition D'une révolution à l'autre.

(13) Bozonnet, J.-P. (dir.), L'écologisme à l'aube du XXIe siècle : de la rupture à la banalisation? Genève : Georg, 2000.

(14) Micoud, A. (avec la collaboration de Charvolin, F. et Regazzola, T.), La dynamique des associations de nature et d'environnement. Ministère de l'Environnement, rapport de synthèse, CRESAL, mars 2000.

(15) Marc Ambroise Rendu, Le Monde, 12 juin 1992.

(16) Gro Harlem Brundtland, chef du gouvernement norvégien, donna en 1987 une définition du développement durable dans un rapport Notre avenir à tous commandé par l'ONU : «Un développement qui répond aux besoins présents sans compromettre la capacité des générations futures à répondre aux leurs".

(17) Voir par exemple les travaux de Gilles Brougère.

(18) Plusieurs jeux d'information et de prévention sur le sida, prenant eux aussi la forme de jeux de l'oie et de Trivial Pursuit, ont été collectés par nos collègues du musée, Françoise Loux et Stéphane Abriol.

(19 Fischler, C. L'homnivore. Le goût, la cuisine et le corps. Paris : Éditions Odile Jacob, 1993

(20) Davallon, J., op. cit., p. 20.

(21) Le Menestrel, S. La collecte de l'objet contemporain. Un défi posé au musée de la Civilisation à Québec, Ethnologie française, tome XXVI, 1996, pp. 74-89. Abriol, S. et Loux, F. La place du sida dans un musée de société, Musées et collections publiques de France, n 237, 2002/2003, pp. 36-41. 